\title{
ON THE OPTIMAL CONTROL OF A CLASS OF HYPERBOLIC SYSTEMS
}

\author{
ZhAN SHeng Wu
}

In this thesis, we consider a class of optimal control problems involving hyperbolic partial differential equations with Darboux boundary conditions. This class of problems was reported in [2] and later on studied extensively by Suryanarayana [4, 5, 6, 7] using the approaches suggested by Cesari [3]. They investigated questions concerning necessary condition for optimality and existence of optimal controls. Note that a different result on the existence theorem for this class of optimal control problems was also obtained by Ahmed and Teo [1].

The aim of this thesis is to devise computational algorithms for solving the optimal control problems under consideration. However, our main emphases are on the mathematical theory underlying the techniques and the convergence properties of the algorithms.

In Chapter I, we present a review of certain known results concerning optimal control problems of distributed parameter systems. Problems of computational algorithms for solving optimal control problems of lumped or distributed parameter systems are also included.

We describe the systems and the corresponding optimal control problems in Chapter II. In addition, a brief review of certain known results concerning the dynamical systems and the corresponding optimal control problems are also included. These results are the preparatory materials for the later chapters of this thesis.

Received I June 1982. Thesis submitted to the University of New South Wales, December 1981. Degree approved, April 1982. Supervisor: Dr K.L. Teo. 
In Chapter III, a linear version of the optimal control problem is considered. More precisely, the optimal control problem consists of a linear dynamical system and a linear cost functional. For this special problem, complete answers to four major questions found in the study of optimal control theory are possible, namely,

(i) necessary condition for optimality,

(ii) sufficient condition for optimality,

(iii) existence of optimal controls,

(iv) method for constructing an optimal control.

In Chapter IV, we consider a class of optimal control problems involving linear dynamical systems and convex cost functionals. For this class of problems, answers to the following three questions are possible:

(i) necessary condition for optimality,

(ii) sufficient condition for optimality,

(iii) existence of optimal controls.

Furthermore, an iterative algorithm based on the results in Chapter III is devised for solving the optimal control problem under discussion. The following convergence properties of the algorithm are also obtained.

(i) Let $\left\{u^{k}\right\}$ be the sequence of the admissible controls generated by the algorithm. Then

$$
J\left(u^{k}\right) \rightarrow J\left(u^{*}\right)
$$

where $J$ is the cost functional and $u^{*}$ is an optimal control.

(ii) $u^{k}$ converges to the optimal control $u^{*}$ in the weak ${ }^{*}$ topology of $L_{\infty}$ and in the almost everywhere topology.

The crucial step in the investigation of these convergence properties is the results obtained for the linear optimal control problem in Chapter III.

In Chapter V, a strong variation algorithm is used to obtain an iterative algorithm for solving a linear optimal control problem in which controls appear not only in the forcing terms but also in some coefficients of the system involved. This optimal control problem contains 
the linear problem of Chapter III as a special case, but overlaps with those considered in Chapter IV. The type of convergence of the algorithm is as follows.

Let $\left\{u^{k}\right\}$ be a sequence of admissible controls obtained by the algorithm. Then, any accumulation point of the sequence $\left\{u^{k}\right\}$ in the $L_{\infty}$ topology, if it exists, satisfies a necessary condition for optimality.

In Chapter VI, the general nonlinear optimal control problem posed in Chapter II is considered. The conditional gradient technique is used to devise an iterative algorithm for solving this general optimal control problem. Furthermore, the same type of convergence property as that of Chapter $\mathrm{V}$ is also obtained.

In Chapter VII, we compare the results obtained in Chapters III to VI. Furthermore, some open problems are also briefly discussed.

\section{References}

[1] Nasin Uddin Ahmed and K.L. Teo, Optimal control of distributed parameter systems (North-Holland, New York, Amsterdam, 1981).

[2] A.G. Butkovskiy, Distributed control systems (Modern Analytic and Computational Methods in Science and Mathematics, 11. American Elsevier, New York, 1969).

[3] Lamberto Cesari, "Optimization with partial differential equations in Dkeudonne-Rashevsky form and conjugate problems", Arch. Rational Mech. Anal. 33 (1969), 339-357.

[4] Mandi Butchi Suryanarayana, "Optimization problems with hyperbolic partial differential equations" ( $\mathrm{PhD}$ thesis, University of Michigan, Ann Arbor, 1970).

[5] M.B. Suryanarayana, "Necessary conditions for optimization problems with hyperbolic partial differential equations", SIAM J. Control 11 (1973), 130-147.

[6] M.B. Suryanarayana, "Existence theorems for optimization problems concerning hyperbolic partial differential equations", J. Optim. Theory Appt. 15 (1975), 361-392. 
[7] M.B. Suryanarayana, "Existence theorems for optimization problems concerning linear, hyperbolic partial differential equations without convexity conditions", J. Optim. Theory Appl. 19 (1976), 47-61.

Department of Mathematics,

Zhongshan University,

Guangzhou,

China. 